\title{
Transforming Fuzzy Description Logics into Classical Description Logics
}

\author{
Umberto Straccia \\ ISTI-CNR, Via G. Moruzzi 1, I-56124 Pisa, ITALY \\ stracciadisti.cnr.it
}

\begin{abstract}
In this paper we consider Description Logics (DLs), which are logics for managing structured knowledge, with a well-known fuzzy extension to deal with vague information. While for fuzzy DLs ad-hoc, tableaux-like reasoning procedures have been given in the literature, the topic of this paper is to present a reasoning preserving transformation of fuzzy DLs into classical DLs. This has the considerable practical consequence that reasoning in fuzzy DLs is feasible using already existing DL systems.
\end{abstract}

\section{Introduction}

In the last decade a substantial amount of work has been carried out in the context of Description Logics (DLs) [1]. DLs are a logical reconstruction of the so-called framebased knowledge representation languages, with the aim of providing a simple wellestablished Tarski-style declarative semantics to capture the meaning of the most popular features of structured representation of knowledge. Nowadays, a whole family of knowledge representation systems has been build using DLs, which differ with respect to their expressiveness and their complexity, and they have been used for building a variety of applications (see the DL community home page http://dl.kr.org/).

Despite their growing popularity, relative little work has been carried out ${ }^{1}$ in extending them to the management of uncertain information. This is a well-known and important issue whenever the real world information to be represented is of imperfect nature. In DLs, the problem has attracted the attention of some researchers and some frameworks have been proposed, which differ in the underlying notion of uncertainty, e.g. probability theory $[10,11,15,18,25]$, possibility theory [13], metric spaces [22], fuzzy theory $[7,12,27,29,30]$ and multi-valued theory $[27,28]$.

In this paper we consider the fuzzy extension of DLs towards the management of vague knowledge [27]. The choice of fuzzy set theory [31] as a way of endowing a DL with the capability to deal with imprecision is motivated as fuzzy logics capture the notion of imprecise concept, i.e. a concept for which a clear and precise definition is not possible. Therefore, fuzzy DLs allow to express that a sentence, like "it is Cold", is not just true or false like in classical DLs, but has a degree of truth, which is taken from the real unit interval $[0,1]$. The truth degree dictates to which extent a sentence is true.

The fuzzy DL we consider ([27]) has been applied in the context of Logic-based Multimedia Information Retrieval (LMIR) $[23,26]$ in which multimedia documents are

\footnotetext{
${ }^{1}$ Comparing with other formalisms -notably logic programming (see, e.g. [17, 20], for an overview).
} 
"semantically annotated" (more generally, the logic is applicable to the context of the Semantic Web [5] as well, where DLs already play an important role [14]). LMIR points out the necessity of extending DLs with capabilities which allow the treatment of the inherent imprecision in multimedia object content representation and retrieval. In fact, classical DLs are insufficient for describing real multimedia retrieval situations, as the retrieval is usually not only a yes-no question: $(i)$ the representations of multimedia objects' content and queries which the system (and the logic) have access to are inherently imperfect; and (ii) the relevance of a multimedia object to a query can thus be established only up to a limited degree (an explanatory example will be provided later one in the paper).

However, from a computational point of view, the reasoning procedures in [27], which are at the core of the LMIR model and system described in [23], are based on an ad-hoc tableaux calculus, similar to the ones presented for almost all DLs. Unfortunately, a drawback of the tableaux calculus in [27] is that any system, which would like to implement this fuzzy logic, has to be worked out from scratch (as we did in [23]) and requires a notable effort to become truly efficient (which was not the case for [23]).

The contribution of this paper is as follows. Primarily, we present a reasoning preserving transformation of fuzzy DLs into classical DLs. This has the considerable practical consequence that reasoning in fuzzy DLs is feasible using already existing DL systems and may take advantage of their efficiency. Secondarily, we allow the representation of so-called general terminological axioms, while in $[23,27]$, the axioms were very limited in the form. To best of our knowledge, no algorithm has yet been worked out for general axioms in fuzzy DLs. Overall, our approach may be extended to more expressive DLs than the one we present here as well and turns out to be very useful for our LMIR model and system.

We proceed as follows. In the next section, we recall some minimal notions about DLs. In Section 3 we recall fuzzy DLs and show their application to LMIR. Section 4 is the main part of this paper, where we present our reduction of fuzzy DLs into classical DLs, while Section 5 concludes.

\section{A quick look to DLs}

Instrumental to our purpose, the specific DL we extend with "fuzzy" capabilities is $\mathcal{A L C}$ with role hierachies, i.e. $\mathcal{A} \mathcal{L C H}$, a significant representative of DLs (see, e.g. [1]). $\mathcal{A L C H}$ is sufficiently expressive to illustrate the main concepts introduced in this paper. More expressive DLs will be the subject of an extended work. Note that $[23,27]$ considered $\mathcal{A L C}$ only. So, consider three alphabets of symbols, for concept names (denoted $A$ ), for role names (denoted $R$ ) and individual names (denoted $a$ and $b)^{2}$. A concept (denoted $C$ or $D$ ) of the language $\mathcal{A L C H}$ is built inductively from concept names $A$, role names $R$, top concept $\top$ and bottom concept $\perp$, according to the following syntax rule:

$$
\begin{aligned}
C, D \longrightarrow & C \sqcap D \mid \text { (concept conjunction) } \\
C & \sqcup D \mid \text { (concept disjunction) } \\
& \neg C \mid \text { (concept negation) } \\
& \forall R . C \mid \text { (universal quantifi cation) } \\
& \exists R . C \text { (existential quantifi cation) . }
\end{aligned}
$$

\footnotetext{
${ }^{2}$ Metavariables may have a subscript or a superscript.
} 
A terminology, $\mathcal{T}$, is a finite set of concept inclusions or role inclusions, called terminological axioms, $\tau$, where given two concepts $C$ and $D$, and two role names $R$ and $R^{\prime}$, a terminological axiom is an expression of the form $C \sqsubseteq D$ ( $D$ subsumes $C$ ) or of the form $R \sqsubseteq R^{\prime}$ ( $R^{\prime}$ subsumes $R$ ). We also write $C=D$ (concept definition) as a short hand for $C \sqsubseteq D$ and $D \sqsubseteq C$ (and similarly for role definitions). An assertion, $\alpha$, is an expression of the form $a: C$ (" $a$ is an instance of $C$ "), or an expression $(a, b): R$ (" $(a, b)$ is an instance of $R$ "). A Knowledge Base $(\mathrm{KB}), \mathcal{K}=\langle\mathcal{T}, \mathcal{A}\rangle$, is such that $\mathcal{T}$ and $\mathcal{A}$ are finite sets of terminological axioms and assertions, respectively.

An interpretation $\mathcal{I}$ is a pair $\mathcal{I}=\left(\Delta^{\mathcal{I}},{ }^{\mathcal{I}}\right)$ consisting of a non empty set $\Delta^{\mathcal{I}}$ (called the domain) and of an interpretation function. ${ }^{\mathcal{I}}$ mapping individuals into elements of $\Delta^{\mathcal{I}}$ (note that usually the unique name assumption ${ }^{3}$ is considered, but it does not matter us here), concepts names into subsets of $\Delta^{\mathcal{I}}$, roles names into subsets of $\Delta^{\mathcal{I}} \times \Delta^{\mathcal{I}}$ and satisfies $\top^{\mathcal{I}}=\Delta^{\mathcal{I}}$ and $\perp^{\mathcal{I}}=\emptyset$. The interpretation of complex concepts is defined inductively as usual:

$$
\begin{aligned}
(C \sqcap D)^{\mathcal{I}} & =C^{\mathcal{I}} \cap D^{\mathcal{I}} \\
(C \sqcup D)^{\mathcal{I}} & =C^{\mathcal{I}} \cup D^{\mathcal{I}} \\
(\neg C)^{\mathcal{I}} & =\Delta^{\mathcal{I}} \backslash C^{\mathcal{I}} \\
(\forall R . C)^{\mathcal{I}} & =\left\{d \in \Delta^{\mathcal{I}} \mid \forall d^{\prime} .\left(d, d^{\prime}\right) \notin R^{\mathcal{I}} \text { or } d^{\prime} \in C^{\mathcal{I}}\right\} \\
(\exists R . C)^{\mathcal{I}} & =\left\{d \in \Delta^{\mathcal{I}} \mid \exists d^{\prime} .\left(d, d^{\prime}\right) \in R^{\mathcal{I}} \text { and } d^{\prime} \in C^{\mathcal{I}}\right\} .
\end{aligned}
$$

A concept $C$ is satisfiable iff there is an interpretation $\mathcal{I}$ such that $C^{\mathcal{I}} \neq \emptyset$. Two concepts $C$ and $D$ are equivalent (denoted $C \equiv D$ ) iff $C^{\mathcal{I}}=D^{\mathcal{I}}$, for all interpretations $\mathcal{I}$. An interpretation $\mathcal{I}$ satisfies an assertion $a: C$ (resp. $(a, b): R)$ iff $a^{\mathcal{I}} \in C^{\mathcal{I}}$ (resp. $\left(a^{\mathcal{I}}, b^{\mathcal{I}}\right) \in R^{\mathcal{I}}$ ), while $\mathcal{I}$ satisfies a terminological axiom $C \sqsubseteq D$ iff $C^{\mathcal{I}} \subseteq D^{\mathcal{I}}$. The satisfiability of role inclusions $R \sqsubseteq R^{\prime}$ is similar. Furthermore, an interpretation $\mathcal{I}$ satisfies (is a model of) a terminology $\mathcal{T}$ (resp. a set of assertions $\mathcal{A}$ ) iff $\mathcal{I}$ satisfies each element in $\mathcal{T}$ (resp. $\mathcal{A}$ ), while $\mathcal{I}$ satisfies (is a model of) a $\mathrm{KB} \mathcal{K}=\langle\mathcal{T}, \mathcal{A}\rangle$ iff $\mathcal{I}$ satisfies both $\mathcal{T}$ and $\mathcal{A}$. Finally, given a $\mathrm{KB} \mathcal{K}$ and an assertion $\alpha$ we say that $\mathcal{K}$ entails $\alpha$, denoted $\mathcal{K}=\alpha$, iff each model of $\mathcal{K}$ satisfies $\alpha$.

Example 1. Consider the following simple $\mathrm{KB}, \mathcal{K}=\langle\mathcal{T}, \mathcal{A}\rangle$, where

$$
\mathcal{T}=\{\text { Bird } \sqsubseteq \text { Animal, Dog } \sqsubseteq \text { Animal }\}, \mathcal{A}=\{\text { snoopy:Dog, woodstock:Bird }\} .
$$

Consider the query concept Animal, i.e. we are looking for animals. It can easily be shown that both Snoopy and Woodstock are animals, i.e. $\{a|\mathcal{K}| a$ :Animal $\}=$ $\{$ snoopy, woodstock\}.

The next example is slightly more involved.

Example 2. Consider the following $\mathrm{KB} \mathcal{K}=\langle\mathcal{T}, \mathcal{A}\rangle$, where

$$
\mathcal{T}=\{A=\forall R . \neg B\}, \mathcal{A}=\{a: \forall R . C\} .
$$

Consider the assertion $\alpha=a: A \sqcup \exists R$. $(B \sqcap C)$. It can be shown that $\mathcal{K} \models \alpha$ holds. In fact, consider a model $\mathcal{I}$ of $\mathcal{K}$. Then either $a^{\mathcal{I}} \in A^{\mathcal{I}}$ or $a^{\mathcal{I}} \notin A^{\mathcal{I}}$. In the former case, $\mathcal{I}$ satisfies $\alpha$. In the latter case, as $\mathcal{I}$ satisfies $\mathcal{T}, a^{\mathcal{I}} \notin(\forall R . \neg B)^{\mathcal{I}}$, i.e. $a^{\mathcal{I}} \in(\exists R . B)^{\mathcal{I}}$ holds. But, $\mathcal{I}$ satisfies $\mathcal{A}$ as well, i.e. $a^{\mathcal{I}} \in(\forall R . C)^{\mathcal{I}}$ and, thus, $a^{\mathcal{I}} \in(\exists R .(B \sqcap C))^{\mathcal{I}}$. Therefore, $\mathcal{I}$ satisfies $\alpha$, which concludes.

$$
{ }^{3} a^{\mathcal{I}} \neq b^{\mathcal{I}} \text {, if } a \neq b \text {. }
$$


Finally, note that there are efficient implemented reasoners like, for instance, RACER ${ }^{4}$ or FACT ${ }^{5}$, which allow to reason in quite more expressive DLs as $\mathcal{A L C H}$.

\section{A quick look to fuzzy DLs}

We recall here the main notions related to fuzzy DLs, taken from [27]. Worth noting is that we deal with general terminological axioms of the form $C \sqsubseteq D$, while in [23, 27] the terminological component is restricted in the form. For convenience, we call the fuzzy extension of $\mathcal{A L C H}, \mathfrak{f} \mathcal{A L C H}$. The main idea underlying $\mathfrak{f} \mathcal{A} \mathcal{L C H}$ is that an assertion $a: C$, rather being interpreted as either true or false, will be mapped into a truth value $c \in[0,1]$. The intended meaning is that $c$ indicates to which extend (how certain it is that) ' $a$ is a $C$ '. Similarly for role names.

Formally, a finterpretation is a pair $\mathcal{I}=\left(\Delta^{\mathcal{I}},{ }^{\mathcal{I}}\right)$, where $\Delta^{\mathcal{I}}$ is the domain and $\cdot^{\mathcal{I}}$ is an interpretation function mapping

- individuals as for the classical case;

- a concept $C$ into a function $C^{\mathcal{I}}: \Delta^{\mathcal{I}} \rightarrow[0,1]$; and

- a role $R$ into a function $R^{\mathcal{I}}: \Delta^{\mathcal{I}} \times \Delta^{\mathcal{I}} \rightarrow[0,1]$.

If $C$ is a concept then $C^{\mathcal{I}}$ will naturally be interpreted as the membership degree function ( $f_{C}$ in 'fuzzy notation') of the fuzzy concept (set) $C$ w.r.t. $\mathcal{I}$, i.e. if $d \in \Delta^{\mathcal{I}}$ is an object of the domain $\Delta^{\mathcal{I}}$ then $C^{\mathcal{I}}(d)$ gives us the degree of being the object $d$ an element of the fuzzy concept $C$ under the finterpretation $\mathcal{I}$. Similarly for roles.

The definition of concept equivalence is like for $\mathcal{A L C H}$. Two concepts $C$ and $D$ are equivalent iff $C^{\mathcal{I}}=D^{\mathcal{I}}$, for all finterpretations $\mathcal{I}$. The interpretation function ${ }^{\mathcal{I}}$ has also to satisfy the following equations: for all $d \in \Delta^{\mathcal{I}}, \top^{\mathcal{I}}(d)=1, \perp^{\mathcal{I}}(d)=0$ and

$$
\begin{aligned}
& (C \sqcap D)^{\mathcal{I}}(d)=\min \left(C^{\mathcal{I}}(d), D^{\mathcal{I}}(d)\right) \\
& (C \sqcup D)^{\mathcal{I}}(d)=\max \left(C^{\mathcal{I}}(d), D^{\mathcal{I}}(d)\right) \\
& (\neg C)^{\mathcal{I}}(d)=1-C^{\mathcal{I}}(d) \\
& (\forall R . C)^{\mathcal{I}}(d)=\inf _{d^{\prime} \in \Delta^{\mathcal{I}}}\left\{\max \left(1-R^{\mathcal{I}}\left(d, d^{\prime}\right), C^{\mathcal{I}}\left(d^{\prime}\right)\right)\right\} \\
& (\exists R . C)^{\mathcal{I}}(d)=\sup _{d^{\prime} \in \Delta^{\mathcal{I}}}\left\{\min \left(R^{\mathcal{I}}\left(d, d^{\prime}\right), C^{\mathcal{I}}\left(d^{\prime}\right)\right)\right\} .
\end{aligned}
$$

These equations are the standard interpretation of conjunction, disjunction, negation and quantification, respectively for fuzzy sets [31] (see also [21,29]). Nonetheless, some conditions deserve an explanation. The semantics of $\exists R . C$ is the result of viewing $\exists R . C$ as the open first order formula $\exists y \cdot R(x, y) \wedge \bar{C}(y)$ (where $\bar{C}$ is the translation of $C$ into first-order logic) and $\exists$ is viewed as a disjunction over the elements of the domain. Similarly, the semantics of $\forall R . C$ is related to $\forall y . \neg R(x, y) \vee \bar{C}(y)$, where $\forall$ is viewed as a conjunction over the elements of the domain. As for the classical DLs, dual relationships between concepts hold: e.g. $(C \sqcap D) \equiv \neg(\neg C \sqcup \neg D)$ and $(\forall R . C) \equiv \neg(\exists R . \neg C)$, but $C \sqcap(\neg C \sqcup D) \not \equiv D$.

A f assertion (denoted $\mathfrak{f} \alpha$ ) is an expression of the form $\left\langle\alpha \geq c_{1}\right\rangle,\left\langle\alpha>c_{2}\right\rangle,\left\langle\alpha^{\prime} \leq c_{2}\right\rangle$ or $\left\langle\alpha^{\prime}<c_{1}\right\rangle$, where $\alpha$ is an $\mathcal{A L C H}$ assertion, $c_{1} \in(0,1]$ and $c_{2} \in[0,1)$, but $\alpha^{\prime}$ is an $\mathcal{A L C H}$ assertion of the form $a: C$ only. For coherence, we do not allow fassertions of the

\footnotetext{
${ }^{4}$ http://www.cs.concordia.ca/ haarslev/racer/

${ }^{5}$ http://www.cs.man.ac.uk/ horrocks/FaCT/
} 
form $\langle(a, b): R \leq c\rangle$ or $\langle(a, b): R<c\rangle$ as they relate to 'negated roles', which is not part of classical $\mathcal{A} \mathcal{L C H}$. From a semantics point of view, a fassertion $\langle\alpha \leq c\rangle$ constrains the truth value of $\alpha$ to be less or equal to $c$ (similarly for $\geq,>$ and $<$ ). So, a finterpretation $\mathcal{I}$ satisfies $\langle a: C \geq c\rangle$ (resp. $\langle(a, b): R \geq c\rangle$ ) iff $C^{\mathcal{I}}\left(a^{\mathcal{I}}\right) \geq c\left(\right.$ resp. $\left.R^{\mathcal{I}}\left(a^{\mathcal{I}}, b^{\mathcal{I}}\right) \geq c\right)$. Similarly for $>, \leq$ and $\langle$. Note that, e.g. $\langle a: \neg C \geq c\rangle$ and $\langle a: C \leq 1-c\rangle$ are satisfied by the same set of finterpretations.

Concerning terminological axioms, a $\mathfrak{f} \mathcal{A L C H}$ terminological axiom is, as for the classical DL $\mathcal{A L C H}$, of the form $C \sqsubseteq D$, where $C$ and $D$ are $\mathcal{A L C H}$ concepts, or of the form $R \sqsubseteq R^{\prime}$, where $R$ and $R^{\prime}$ are role names. From a semantics point of view, a finterpretation $\mathcal{I}$ satisfies $C \sqsubseteq D$ iff for all $d \in \Delta^{\mathcal{I}}, C^{\mathcal{I}}(d) \leq D^{\mathcal{I}}(d)$. Similarly, finterpretation $\mathcal{I}$ satisfies $R \sqsubseteq \overline{R^{\prime}}$ iff for all $\left\{d, d^{\prime}\right\} \subseteq \Delta^{\mathcal{I}}, R^{\mathcal{I}}\left(d, \overline{d^{\prime}}\right) \leq R^{\prime \mathcal{I}}\left(d, d^{\prime}\right)$.

A $\mathfrak{f}$ Knowledge Base $(\mathfrak{f K B})$ is pair $\mathfrak{f} \mathcal{K}=\langle\mathcal{T}, \mathcal{A}\rangle$, where $\mathcal{T}$ and $\mathcal{A}$ are finite sets of terminological axioms and fassertions, respectively. A finterpretation $\mathcal{I}$ satisfies (is a model of) a terminology $\mathcal{T}$ (resp. a set of fassertions $\mathcal{A}$ ) iff $\mathcal{I}$ satisfies each element in $\mathcal{T}$ (resp. $\mathcal{A}$ ), while $\mathcal{I}$ satisfies (is a model of) a $\mathrm{KB} \mathfrak{f} \mathcal{K}=\langle\mathcal{T}, \mathcal{A}\rangle$ iff $\mathcal{I}$ satisfies both $\mathcal{T}$ and $\mathcal{A}$. Given a $\mathfrak{f K B} \mathfrak{f} \mathcal{K}$, and a fassertion $\mathfrak{f} \alpha$, we say that $\mathfrak{f} \mathcal{K}$ entails $\mathfrak{f} \alpha$, denoted $\mathfrak{f} \mathcal{K}=\mathfrak{f} \alpha$, iff each model of $\mathfrak{f} \mathcal{K}$ satisfies $\mathfrak{f} \alpha$. For instance, if $c^{\prime}>1-c$ then

$$
\left\{\left\langle(a, b): R \geq c^{\prime}\right\rangle,\langle a: \forall R . C \geq c\rangle\right\} \models\langle b: C \geq c\rangle .
$$

Finally, given $\mathfrak{f} \mathcal{K}$ and an $\mathcal{A L C H}$ assertion $\alpha$, it is of interest to compute $\alpha$ 's best lower and upper truth value bounds. The greatest lower bound of $\alpha$ w.r.t. $\mathfrak{f} \mathcal{K}$ (denoted $g l b(\mathfrak{f} \mathcal{K}, \alpha))$ is $g l b(\mathfrak{f} \mathcal{K}, \alpha)=\sup \{c \mid \mathfrak{f} \mathcal{K} \models\langle\alpha \geq c\rangle\}$, while the least upper bound of $\alpha$ with respect to $\mathfrak{f} \mathcal{K}$ (denoted $\operatorname{lub}(\mathfrak{f} \mathcal{K}, \alpha)$ is $\operatorname{lu} b(\mathfrak{f} \mathcal{K}, \alpha)=\inf \{c \mid \mathfrak{f} \mathcal{K} \models\langle\alpha \leq c\rangle\}$, where $\sup \emptyset=0$ and $\inf \emptyset=1$. Determining the $l u b$ and the $g l b$ is called the Best Truth Value Bound (BTVB) problem. Note that

$$
\operatorname{lub}(\Sigma, a: C)=1-\operatorname{glb}(\Sigma, a: \neg C),
$$

i.e. the $l u b$ can be determined through the $g l b$ (and vice-versa). The same reduction to $g l b$ does not hold for $l u b(\Sigma,(a, b): R)$ as $(a, b): \neg R$ is not an expression of our language. ${ }^{6}$ Finally, note that, $\Sigma \models_{\mathcal{L}}\langle\alpha \geq n\rangle$ iff $\operatorname{glb}(\Sigma, \alpha) \geq n$, and similarly $\Sigma \models_{\mathcal{L}}$ $\langle\alpha \leq n\rangle$ iff $\operatorname{lub}(\Sigma, \alpha) \leq n$ hold. Concerning roles, note that $\Sigma \models_{\mathcal{L}}\langle(a, b): R \geq n\rangle$ iff $\langle(a, b): R \geq m\rangle \in \Sigma$ with $m \geq n$. Therefore,

$$
g l b(\Sigma, R(a, b))=\max \{n \mid\langle R(a, b) \geq n\rangle \in \Sigma\} .
$$

Concerning the entailment problem, it is quite easily verified that the entailment problem can be reduced to the unsatisfiability problem:

$$
\begin{aligned}
& \langle\mathcal{T}, \mathcal{A}\rangle=\langle\alpha \geq n\rangle \text { iff }\langle\mathcal{T}, \mathcal{A} \cup\{\langle\alpha<n\rangle\}\rangle \text { is not satisfi able } \\
& \langle\mathcal{T}, \mathcal{A}\rangle \models\langle\alpha \leq n\rangle \text { iff }\langle\mathcal{T}, \mathcal{A} \cup\{\langle\alpha>n\rangle\}\rangle \text { is not satisfi able }
\end{aligned}
$$

In [27] decision procedures for the satisfiability, the entailment and the BTVB problem are given for $\mathfrak{f} \mathcal{A L C H}$, but with the already discussed restrictions on the form of terminological axioms and terminologies.

\footnotetext{
${ }^{6}$ Of course, $\operatorname{lu} b(\Sigma,(a, b): R)=1-g l b(\Sigma,(a, b): \neg R)$ holds, where $(\neg R)^{\mathcal{I}}\left(d, d^{\prime}\right)=1-$ $R^{\mathcal{I}}\left(d, d^{\prime}\right)$.
} 
Example 3. Similarly to Example 2, consider $\mathfrak{f} \mathcal{K}=\langle\mathcal{T}, \mathcal{A}\rangle$, where

$$
\mathcal{T}=\{A=\forall R . \neg B\}, \mathcal{A}=\{\langle a: \forall R . C \geq 0.7\rangle\} .
$$

Consider the assertion $\alpha=a: A \sqcup \exists R .(B \sqcap C)$. It can be shown that $g l b(\mathfrak{f K}, \alpha)=0.5$ and $\operatorname{lub}(\mathfrak{f} \mathcal{K}, \alpha)=1$ hold. In fact, for any model $\mathcal{I}$ of $\mathfrak{f} \mathcal{K}$, we have that

$$
(A \sqcup \exists R .(B \sqcap C))^{\mathcal{I}}\left(a^{\mathcal{I}}\right) \geq \max (c, \min (0.7,1-c)),
$$

for any $c \in[0,1]$. Indeed, let $\mathcal{I}$ be a model of $\mathfrak{f} \mathcal{K}$. Assume that $(A \sqcup \exists R .(B \sqcap C))^{\mathcal{I}}\left(a^{\mathcal{I}}\right)=$ $w$. Consider $c \in[0,1]$. Then either $A^{\mathcal{I}}\left(a^{\mathcal{I}}\right) \geq c$ or $A^{\mathcal{I}}\left(a^{\mathcal{I}}\right)<c$. In the former case, it follows that $w \geq c$. In the latter case, as $\mathcal{I}$ satisfies $\mathcal{T}$, from $A^{\mathcal{I}}\left(a^{\mathcal{I}}\right)<c$ it follows that $(\forall R . \neg B)^{\mathcal{I}}\left(a^{\mathcal{I}}\right)<c$. But, $\forall R . \neg B \equiv \neg \exists R . B$ and, thus, $(\exists R . B)^{\mathcal{I}}\left(a^{\mathcal{I}}\right)>$ $1-c$. Therefore, there is $d \in \Delta^{\mathcal{I}}$ such that $R^{\mathcal{I}}\left(a^{\mathcal{I}}, d\right)>1-c$ and $B^{\mathcal{I}}(d)>$ $1-c$. But, $\mathcal{I}$ satisfies $\mathcal{A}$, i.e. $(\forall R . C)^{\mathcal{I}}\left(a^{\mathcal{I}}\right) \geq 0.7$. By definition, this means that $\inf _{d^{\prime} \in \Delta^{\mathcal{I}}}\left\{\max \left(1-R^{\mathcal{I}}\left(a^{\mathcal{I}}, d^{\prime}\right), C^{\mathcal{I}}\left(d^{\prime}\right)\right)\right\} \geq 0.7$ and, in particular, for $d^{\prime}=d, \max (1-$ $\left.R^{\mathcal{I}}\left(a^{\mathcal{I}}, d\right), C^{\mathcal{I}}(d)\right) \geq 0.7$ holds. Therefore, $1-R^{\mathcal{I}}\left(a^{\mathcal{I}}, d\right)<0.7$ (i.e., $R^{\mathcal{I}}\left(a^{\mathcal{I}}, d\right)>0.3$ ) implies $\left.C^{\mathcal{I}}(d)\right) \geq 0.7$. As a consequence, from $R^{\mathcal{I}}\left(a^{\mathcal{I}}, d\right)>1-c$, for $c \leq 0.7$ it follows that $C^{\mathcal{I}}(d) \geq 0.7$ (see also Equation 1). Therefore, $(\exists R .(B \sqcap C))^{\mathcal{I}}\left(a^{\mathcal{I}}\right) \geq$ $\min (0.7,1-c)$ and, thus, $w \geq \max (c, \min (0.7,1-c))$, which proofs $(6)$. Finally, as for any $c \in[0,1], \max (c, \min (0.7,1-c)) \geq 0.5$ and there is no $c^{\prime}>0.5$ such that for all $c \in[0,1], \max (c, \min (0.7,1-c)) \geq c^{\prime}$, by $(6), g l b(\mathfrak{f} \mathcal{K}, \alpha)=0.5$ follows.

The proof of $\operatorname{lub}(\mathfrak{f} \mathcal{K}, \alpha)=1$ is easy.

In the following, we show the application of $\mathfrak{f} \mathcal{A L C H}$ to LMIR, which, among others, shows how fassertions are generated automatically.

Logic-based multimedia information retrieval. Let us first roughly present (parts of) the LMIR model of $[23,26]$. In doing this, we rely on Figure 1. The model has two layers addressing the multidimensional aspect of multimedia objects $o \in \mathbb{O}$ (e.g. objects $\circ 1$ and $\circ 2$ in Figure 1): that is, their form and their semantics (or meaning). The form of a multimedia object is a collective name for all its media dependent, typically automatically extracted features, like text index term weights (object of type text), colour distribution, shape, texture, spatial relationships (object of type image), mosaiced videoframe sequences and time relationships (object of type video). On the other hand, the semantics (or meaning) of a multimedia object is a collective name for those features that pertain to the slice of the real world being represented, which exists independently of the existence of a object referring to it. Unlike form, the semantics of a multimedia object is thus media independent (typically, constructed manually perhaps with the assistance of some automatic tool). Therefore, we have two layers, the object form layer and the object semantics layer. The former represents media dependent features of the objects, while the latter describes the semantic properties of the slice of world the objects are about. The semantic entities (e.g., Snoopy, Woodstock), which objects can be about are called semantic index terms $(t \in \mathbb{T})$. The mapping of objects $o \in \mathbb{O}$ to semantic entities $t \in \mathbb{T}$ (e.g., "object $\circ 1$ is about Snoopy") is called semantic annotation. According to the fuzzy information retrieval model [6, 16, 19,24], semantic annotation can be formalized as a membership function $F: \mathbb{O} \times \mathbb{T} \rightarrow[0,1]$ describing the correlation between multimedia objects and semantic index terms. The value $F(o, t)$ indicates 


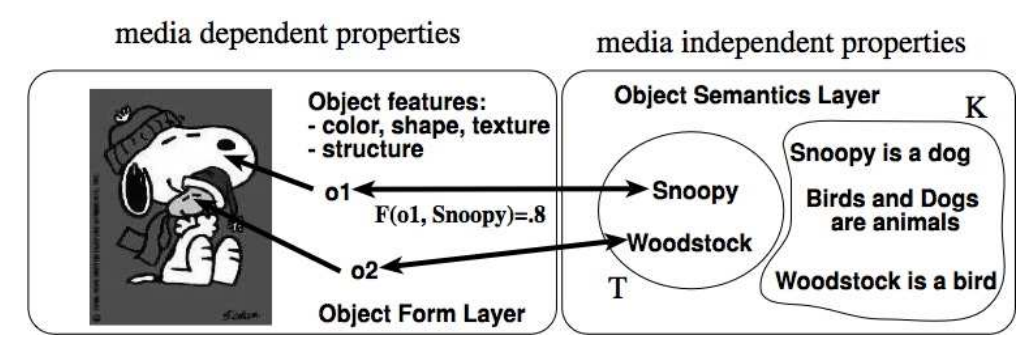

Fig. 1. LMIR model layers and objects

to which degree the multimedia object $o$ deals with the semantic index term $t$. The meaning of a semantic index term $t$ may in this context be represented as a fuzzy subset of multimedia objects in $\mathbb{O}, m(t)$, with the quantitative measure of aboutness being the values of function $F$ for a given semantic index term $t$, i.e. $m(t)=\left\{\left\langle o, f_{t}(o)\right\rangle: o \in \mathbb{O}\right\}$, in which $f_{t}(o)=F(o, t) . m(t)$ is the meaning of term $t$. The function $F$ acts as the membership function of $m(t)$. Depending on the context, the function $F$ may be computed automatically (e.g., for text we may have [8], for images we may have an automated image annotation (classification) tool, as e.g. [9]). Note that the function $F$ will be a source for fassertions of the form $\langle(o, t): F \geq F(o, t)\rangle$ (see [23]). In practice, the scenario depicted in Figure 1 may roughly be represented in $\mathfrak{f} \mathcal{A L C H}$ with the following knowledge base $\mathfrak{f} \mathcal{K}=\langle\mathcal{T}, \mathcal{A}\rangle$ :

$$
\begin{aligned}
\mathcal{T}= & \{\text { Bird } \sqsubseteq \text { Animal }, \text { Dog } \sqsubseteq \text { Animal, Object } \sqcap \text { IndexTerm } \sqsubseteq \perp, F=\text { IsAbout }\} \\
\mathcal{A}= & \{\langle\text { snoopy:Dog } \geq 1\rangle,\langle\text { woodstock:Bird } \geq 1\rangle, \\
& \langle(\text { o1, snoopy }): \mathrm{F} \geq 0.8\rangle,\langle(\text { o2, woodstock }): \mathrm{F} \geq 0.7\rangle\} .
\end{aligned}
$$

Now, consider the query concept $Q=$ Object. $\exists$ IsAbout.Animal, i.e. retrieve all objects about animals. Then it is easily verified that we retrieve both objects, but with different Retrieval Status Values [2], which indicate their relatedness to the query $(\mathcal{K}=$ $\langle$ snoopy: $Q \geq 0.8\rangle, \mathcal{K} \models\langle$ woodstock: $Q \geq 0.7\rangle$ ).

\section{Mapping $\mathfrak{f} \mathcal{A L C H}$ into $\mathcal{A L C H}$}

Our aim is to map $\mathfrak{f} \mathcal{A L C H}$ knowledge bases into satisfiability and entailment preserving classical $\mathcal{A L C H}$ knowledge bases. An immediate consequence is then that $(i)$ we have reasoning procedures for $\mathfrak{f} \mathcal{A L C H}$ with general terminological axioms, which are still unknown; and $(i i)$ we can rely on already implemented reasoners to reason in $\mathfrak{f} \mathcal{A L C H}$.

Before we are going to formally present the mapping, we first illustrate the basic idea we rely on. Our mapping relies on ideas presented in $[3,4]$ for so-called regular multi-valued logics.

Assume we have a $\mathfrak{f K B}, \mathfrak{f} \mathcal{K}=\langle\emptyset, \mathcal{A}\rangle$, where $\mathcal{A}=\left\{\mathfrak{f} \alpha_{1}, \mathfrak{f} \alpha_{2}, \mathfrak{f} \alpha_{3}, \mathfrak{f} \alpha_{4}\right\}$ and $\mathfrak{f} \alpha_{1}=$ $\langle a: A \geq 0.4\rangle, \mathfrak{f} \alpha_{2}=\langle a: A \leq 0.7\rangle, \mathfrak{f} \alpha_{3}=\langle a: B \leq 0.2\rangle, \mathfrak{f} \alpha_{4}=\langle b: B \leq 0.1\rangle$. Let us introduce some new concepts, namely $A_{\geq 0.4}, A_{\leq 0.7}, B_{\leq 0.2}$ and $B_{\leq 0.1}$. Informally, the concept $A_{\geq 0.4}$ represents the set of individuals, which are instance of $A$ with degree $c \geq 0.4$, while $A_{\leq 0.7}$ represents the set of individuals, which are instance of $A$ with 
degree $c \leq 0.7$. Similarly, for the other concepts. Of course, we have to consider also the relationships among the introduced concepts. For instance, we need the terminological axiom $B_{\leq 0.1} \sqsubseteq B_{\leq 0.2}$. This axiom dictates that if a truth value is $\leq 0.1$ then it is also $\leq 0.2$. We may represent, thus, the fassertion $\mathfrak{f} \alpha_{1}$ with the $\mathcal{A L C H}$ assertion $a: A_{\geq 0.4}$, indicating that $a$ is an instance of $A$ with a degree $\geq 0.4$. Similarly, $\mathfrak{f} \alpha_{2}$ may be mapped into $a: A_{\geq 0.7}, \mathfrak{f} \alpha_{3}$ may be mapped into $a: B_{\geq 0.2}$, while $\mathfrak{f} \alpha_{4}$ may be mapped into $b: B_{\geq 0.1}$. From a semantics point of view, let us consider the so-called canonical model [1] $\mathcal{I}$ of the resulting classical $\mathcal{A L C H} \mathrm{KB}$, i.e.

$$
\mathcal{I}=\left\{A_{\geq 0.4}(a), A_{\leq 0.7}(a), B_{\leq 0.2}(a), B_{\leq 0.1}(b), B_{\leq 0.2}(b)\right\} .
$$

It is then easily verified that, from $\mathcal{I}$ a model $\mathcal{I}^{\prime}$ of $\mathfrak{f} \mathcal{K}$ can easily be built and, vice-versa, if $\mathcal{I}^{\prime}$ is a model of $\mathfrak{f} \mathcal{K}$, then a model like $\mathcal{I}$ above can be obtained as well. Therefore, our transformation of $\mathfrak{f} \mathcal{K}$ into an $\mathcal{A L C H} \mathrm{KB}$, at least for the above case, is satisfiability preserving. This illustrates our basic idea.

Let us now proceed formally. Consider a $\mathfrak{f K B} \mathfrak{f K}=\langle\mathcal{T}, \mathcal{A}\rangle$. Let $\mathcal{A}^{\mathfrak{f} \mathcal{K}}$ and $\mathcal{R}^{\mathfrak{f} \mathcal{K}}$ be the set of concept names and concept roles occurring in $\mathfrak{f} \mathcal{K}$. Of course, both $\left|\mathcal{A}^{\mathfrak{f} \mathcal{K}}\right|$ and $\left|\mathcal{R}^{\mathfrak{f} \mathcal{K}}\right|$ are linearly bounded by $|\mathfrak{f} \mathcal{K}|$. Consider

$$
\begin{aligned}
X^{\mathfrak{f}}= & \{0,0.5,1\} \cup\{c \mid\langle\alpha \geq c\rangle \in \mathcal{A}\} \\
& \cup\{c \mid\langle\alpha>c\rangle \in \mathcal{A}\} \\
& \cup\{1-c \mid\langle\alpha \leq c\rangle \in \mathcal{A}\} \\
& \cup\{1-c \mid\langle\alpha<c\rangle \in \mathcal{A}\}
\end{aligned}
$$

from which we define

$$
N^{\mathfrak{f} \mathcal{K}}=X^{\mathfrak{f} \mathcal{K}} \cup\left\{1-c \mid c \in X^{\mathfrak{f} \mathcal{K}}\right\}
$$

Note that $\left|N^{\mathfrak{f} \mathcal{K}}\right|$ is linearly bounded by $|\mathcal{A}|$. Essentially, with $N^{\mathfrak{f} \mathcal{K}}$ we collect from $\mathfrak{f} \mathcal{K}$ all the relevant numbers we require for the transformation. Without loss of generality, we may assume that $N^{\mathfrak{f} \mathcal{K}}=\left\{c_{1}, \ldots, c_{\left|N_{\mathcal{f}}\right|}\right\}$ and $c_{i}<c_{i+1}$, for $1 \leq i \leq\left|N^{\mathfrak{f} \mathcal{K}}\right|-1$.

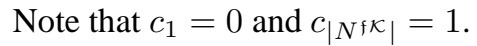

For each $c \in N^{\mathfrak{f K}}$, for each relation $\bowtie \in\{\geq,>, \leq,<\}$, for each $A \in \mathcal{A}^{\mathfrak{f K}}$ and for each $R \in \mathcal{R}^{\mathfrak{f} \mathcal{K}}$, consider a new concept name $A_{\bowtie c}$ and new role names $R_{\geq c}$ and $R_{>c}$, but we do not consider $A_{<0}, A_{>1}$ and $R_{>1}$ (which are not needed). There are as many as $\left(4\left|N^{\mathfrak{f} \mathcal{K}}\right|-2\right)\left|\mathcal{A}^{\mathfrak{f} \mathcal{K}}\right|$ new concept names and $\left(2\left|N^{\mathfrak{f} \mathcal{K}}\right|-1\right)\left|\mathcal{R}^{\mathfrak{f} \mathcal{K}}\right|$ new role names. Note that we do not require new role names $R_{\leq c}$ and $R_{<c}$, as e.g. expressions of the form $\langle(a, b): R \leq c\rangle$ are not part of our language.

Let $\mathcal{T}\left(N^{\mathfrak{f} \mathcal{K}}\right)$ be the following terminology relating the newly introduced concept names and role names: $\mathcal{T}\left(N^{\mathfrak{f} \mathcal{K}}\right)$ is the smallest terminology such that for each $1 \leq i \leq$ $\left|N^{\mathfrak{f} \mathcal{K}}\right|-1$, for each $2 \leq j \leq\left|N^{\mathfrak{f} \mathcal{K}}\right|$, for each $A \in \mathcal{A}^{\mathfrak{f} \mathcal{K}}$ and for each $R \in \mathcal{R}^{\mathfrak{f K}}, \mathcal{T}\left(N^{\mathfrak{f} \overline{\mathcal{K}}}\right)$ contains

$$
\begin{array}{rlrl}
A_{\geq c_{i+1}} & \sqsubseteq A_{>c_{i}} & , & A_{>c_{i}} \sqsubseteq A_{\geq c_{i}} \\
A_{<c_{j}} & \sqsubseteq A_{\leq c_{j}} & , & A_{\leq c_{i}} \sqsubseteq A_{<c_{i+1}} \\
A_{\geq c_{j}} \sqcap A_{<c_{j}} & \sqsubseteq \perp & , A_{>c_{i}} \sqcap A_{\leq c_{i}} \sqsubseteq \perp \\
\top & \sqsubseteq A_{\geq c_{j}} \sqcup A_{<c_{j}}, & \top \sqsubseteq A_{>c_{i}} \sqcup A_{\leq c_{i}} .
\end{array}
$$


The first group reflects the $\geq,<, \leq,>$ ordering among the newly introduced concepts, while the second group identifies 'disjointness' conditions. For instance, among these terminological axioms we may have $A_{\geq 0.4} \sqcap A_{<0.4} \sqsubseteq \perp$ indicating that it cannot be that an individual $a$ is an instance of the concept name $A$ both with degree $\geq 0.4$ and degree $<0.4$. The last group establishes the complimentarily relationships among the new concepts, e.g. $A_{\geq 0.4} \sqcup A_{<0.4} \equiv \top$. Note that $\mathcal{T}\left(N^{\mathfrak{f} \mathcal{K}}\right)$ contains $8\left|\mathcal{A}^{\mathfrak{f} \mathcal{K}}\right|\left(\left|N^{\mathfrak{f} \mathcal{K}}\right|-1\right)$ terminological axioms involving the newly introduced concepts names.

The terminological axioms in $\mathcal{T}\left(N^{\mathfrak{f} \mathcal{K}}\right)$ relating the newly introduced role names are quite similar to the above axioms:

$$
R_{\geq c_{i+1}} \sqsubseteq R_{>c_{i}}, R_{>c_{i}} \sqsubseteq R_{\geq c_{i}} .
$$

Note that $\mathcal{T}\left(N^{\mathfrak{f} \mathcal{K}}\right)$ contains $2\left|\mathcal{R}^{\mathfrak{f} \mathcal{K}}\right|\left(\left|N^{\mathfrak{f} \mathcal{K}}\right|-1\right)$ terminological axioms involving the newly introduced role names. Please note also that in case we would like to allow expressions of the form $\langle(a, b): R \leq c\rangle$ and $\langle(a, b): R<c\rangle$, then we need new role names $R_{\leq c}$ and $R_{<c}$ (excluding $R_{<0}$ ), and terminological axioms $R_{<c_{j}} \sqsubseteq R_{\leq c_{j}}, R_{\leq c_{i}} \sqsubseteq$ $R_{<c_{i+1}}, R_{\geq c_{j}} \sqcap R_{<c_{j}} \sqsubseteq \perp^{r}, R_{>c_{i}} \sqcap R_{\leq c_{i}} \sqsubseteq \perp^{r}, \top^{r} \sqsubseteq R_{\geq c_{j}} \sqcup R_{<c_{j}}$ and $\top^{r} \sqsubseteq$ $R_{>c_{i}} \sqcup R_{\leq c_{i}}$. In particular, note that 'role conjunction', 'role disjunction' and a 'bottom role' and a 'top role' are needed.

Example 4. Consider Example 3. Then $N^{\mathfrak{f} \mathcal{K}}$ is $N^{\mathfrak{f} \mathcal{K}}=\{0,0.3,0.5,0.7,1\}$, while $\mathcal{A}^{\mathfrak{f} \mathcal{K}}=$ $\{A, B, C\}$ and $\mathcal{R}^{\mathfrak{f} \mathcal{K}}=\{R\}$. Below, we provide an excerpt of the terminology $\mathcal{T}\left(N^{\mathfrak{f} \mathcal{K}}\right)$ :

$$
\begin{aligned}
& \mathcal{T}\left(N^{\mathfrak{f} \mathcal{K}}\right)=\left\{A_{\geq 1} \sqsubseteq A_{>0.7}, A_{\geq 0.7} \sqsubseteq A_{>0.5}, \ldots\right\} \\
& \cup\left\{\ldots, A_{\geq 0.3} \sqcap A_{<0.3} \sqsubseteq \perp, \ldots\right\} \\
& \cup\left\{\ldots, \top \sqsubseteq A_{\geq 0.3} \sqcup A_{<0.3}, \ldots\right\} \\
& \cup\left\{\ldots, B_{>1} \sqsubseteq B_{>0.7}, \ldots\right\} \\
& \cup\left\{\ldots, R_{\geq 1} \sqsubseteq R_{>0.7}, \ldots\right\} \text {. }
\end{aligned}
$$

This concludes the management of the newly introduced concept names and role names.

We proceed now with the mapping of the fassertions in a fKB into $\mathcal{A L C H}$ assertions. We define two mappings $\sigma$ and $\rho$, defined as follows. Let $\mathfrak{f} \alpha$ be a fassertion. Then $\sigma$ maps a fassertion into a classical $\mathcal{A L C H}$ assertion, using $\rho$, as follows. In the following, we assume that $c \in[0,1]$ and $\bowtie \in\{\geq,>, \leq,<\}$.

$$
\sigma(\mathfrak{f} \alpha)= \begin{cases}a: \rho(C, \bowtie c) & \text { if } \mathfrak{f} \alpha=\langle a: C \bowtie c\rangle \\ (a, b): \rho(R, \bowtie c) & \text { if } \mathfrak{f} \alpha=\langle(a, b): R \bowtie c\rangle .\end{cases}
$$

We extend $\sigma$ to a set of fassertions $\mathcal{A}$ point-wise, i.e. $\sigma(\mathcal{A})=\{\sigma(\mathfrak{f} \alpha) \mid \mathfrak{f} \alpha \in \mathcal{A}\}$.

The mapping $\rho$ encodes the idea we have previously presented in a simplified example and is inductively defined on the structure of concepts and roles. For roles, we have simply

$$
\rho(R, \bowtie c)=R_{\bowtie c} .
$$

So, for instance the fassertion $\langle(a, b): R \geq c\rangle$ is mapped into the $\mathcal{A L C H}$ assertion $(a, b): R_{\geq c}$. Concerning concepts, we have the following inductive definitions: for $\top$

$$
\rho(\top, \bowtie c)=\left\{\begin{array}{l}
\top \text { if } \bowtie c=\geq c \\
\top \text { if } \bowtie c=>c, c<1 \\
\perp \text { if } \bowtie c=>1 \\
\top \text { if } \bowtie c=\leq 1 \\
\perp \text { if } \bowtie c=\leq c, c<1 \\
\perp \text { if } \bowtie c=<c .
\end{array}\right.
$$


For $\perp$,

For concept name $A$,

$$
\rho(\perp, \bowtie c)=\left\{\begin{array}{l}
\top \text { if } \bowtie c=\geq 0 \\
\perp \text { if } \bowtie c=\geq c, c>0 \\
\perp \text { if } \bowtie c=>c \\
\top \text { if } \bowtie c=\leq c \\
\top \text { if } \bowtie c=<c, c>0 \\
\perp \text { if } \bowtie c=<0 .
\end{array}\right.
$$

For concept conjunction $C \sqcap D$,

$$
\rho(C \sqcap D, \bowtie c)=\left\{\begin{array}{l}
\rho(C, \bowtie c) \sqcap \rho(D, \bowtie c) \text { if } \bowtie \in\{\geq,>\} \\
\rho(C, \bowtie c) \sqcup \rho(D, \bowtie c) \text { if } \bowtie \in\{\leq,<\} .
\end{array}\right.
$$

For concept disjunction $C \sqcup D$,

$$
\rho(C \sqcup D, \bowtie c)=\left\{\begin{array}{l}
\rho(C, \bowtie c) \sqcup \rho(D, \bowtie c) \text { if } \bowtie \in\{\geq,>\} \\
\rho(C, \bowtie c) \sqcap \rho(D, \bowtie c) \text { if } \bowtie \in\{\leq,<\} .
\end{array}\right.
$$

For concept negation $\neg C$,

$$
\rho(\neg C, \bowtie c)=\rho(C, \neg \bowtie 1-c) .
$$

where $\neg \geq=\leq, \neg<=>, \neg \leq=\geq$ and $\neg<=>$. For instance, the fassertion $\langle a: \neg C \geq c\rangle$ is mapped into the $\mathcal{A L C H}$ assertion $a: C_{\leq 1-c}$.

For existential quantification $\exists R . C$,

$$
\rho(\exists R . C, \bowtie c)=\left\{\begin{array}{l}
\exists \rho(R, \bowtie c) \cdot \rho(C, \bowtie c) \text { if } \bowtie \in\{\geq,>\} \\
\forall \rho(R,-\bowtie c) \cdot \rho(C, \bowtie c) \text { if } \bowtie \in\{\leq,<\}
\end{array} .\right.
$$

where $-\leq=>$ and $-<=\geq$. For instance, the fassertion $\langle a: \exists R . C \geq c\rangle$ is mapped into the $\mathcal{A L C H}$ assertion $a: \exists R_{\geq c} . C_{\geq c}$, while $\langle a: \exists R . C \leq c\rangle$ is mapped into $a: \forall R_{>c} . C_{\leq c}$.

Finally, for universal quantification $\forall R . C$,

$$
\rho(\forall R . C, \bowtie c)=\left\{\begin{array}{l}
\forall \rho(R,+\bowtie 1-c) \cdot \rho(C, \bowtie c) \text { if } \bowtie \in\{\geq,>\} \\
\exists \rho(R, \neg \bowtie 1-c) \cdot \rho(C, \bowtie c) \text { if } \bowtie \in\{\leq,<\} .
\end{array}\right.
$$

where $+\geq=>$ and $+>=\geq$. For instance, the fassertion $\langle a: \forall R . C \geq 0.7\rangle$ in Example 3 is mapped into the $\mathcal{A L C H}$ assertion $a: \forall R_{>0.3} . C_{\geq 0.7}$, while $\langle a: \forall R . C \leq c\rangle$ is mapped into $a: \exists R_{\geq 1-c} . C_{\leq c}$.

It is easily verified that for a set of fassertions $\mathcal{A},|\sigma(\mathcal{A})|$ is linearly bounded by $|\mathcal{A}|$.

We conclude with the reduction of a terminological axiom $\tau$ in a terminology $\mathcal{T}$ of a $\mathfrak{f K B} \mathfrak{f} \mathcal{K}=\langle\mathcal{T}, \mathcal{A}\rangle$ into a $\mathcal{A} \mathcal{L C H}$ terminology, $\kappa(\mathfrak{f} \mathcal{K}, \tau)$. Note that a terminological axiom in $\mathfrak{f} \mathcal{A} \mathcal{L C H}$ is reduced into a set of $\mathcal{A L C H}$ terminological axioms. As for $\sigma$, we extend $\kappa$ to a terminology $\mathcal{T}$ point-wise, i.e. $\kappa(\mathfrak{f} \mathcal{K}, \mathcal{T})=\cup_{\tau \in \mathcal{T}} \kappa(\mathfrak{f} \mathcal{K}, \tau) . \kappa(\mathfrak{f} \mathcal{K}, \tau)$ is defined as follows. For a concept specialization $C \sqsubseteq D$,

$$
\begin{aligned}
& \kappa(C \sqsubseteq D)=\bigcup_{c \in N^{\mathcal{K}} \mathcal{K}, \bowtie \in\{\geq,>\}}\{\rho(C, \bowtie c) \sqsubseteq \rho(D, \bowtie c)\} \\
& \bigcup_{c \in N^{f \mathcal{K}}, \bowtie \in\{\leq,<\}}\{\rho(D, \bowtie c) \sqsubseteq \rho(C, \bowtie c)\} .
\end{aligned}
$$

For instance, by relying on the $\mathfrak{f K B} \mathfrak{f} \mathcal{K}$ in Example 3, it can be verified that $\kappa(\mathfrak{f} \mathcal{K}, \mathcal{T})$ contains the $\mathcal{A L C H}$ terminological axioms (e.g. for $c=0.3) A_{\geq 0.3} \sqsubseteq \forall R_{>0.7} . B_{\leq 0.7}$ and $\exists R_{\geq 0.7} \cdot B_{\geq 0.7} \sqsubseteq A_{\leq 0.3}$. 
For a role specialization $R \sqsubseteq R^{\prime}$,

$$
\kappa\left(R \sqsubseteq R^{\prime}\right)=\bigcup_{c \in N^{\mathcal{H}}, \bowtie \in\{\geq,>\}}\left\{\rho(R, \bowtie c) \sqsubseteq \rho\left(R^{\prime}, \bowtie c\right)\right\} .
$$

Note that $|\kappa(\mathfrak{f} \mathcal{K}, \mathcal{T})|$ contains at most $6|\mathcal{T}|\left|N^{\mathfrak{f} \mathcal{K}}\right|$ terminological axioms.

We have now all the ingredients to complete the reduction of a fKB into an $\mathcal{A L C H} \mathrm{KB}$. Let $\mathfrak{f} \mathcal{K}=\langle\mathcal{T}, \mathcal{A}\rangle$ be $\mathfrak{f K B}$. The reduction of $\mathfrak{f} \mathcal{K}$ into an $\mathcal{A} \mathcal{L C H} \mathrm{KB}$, denoted $\mathcal{K}(\mathfrak{f} \mathcal{K})$, is defined as

$$
\mathcal{K}(\mathfrak{f} \mathcal{K})=\left\langle\mathcal{T}\left(N^{\mathfrak{f} \mathcal{K}}\right) \cup \kappa(\mathfrak{f} \mathcal{K}, \mathcal{T}), \sigma(\mathcal{A})\right\rangle .
$$

Therefore, $|\mathcal{K}(\mathfrak{f} \mathcal{K})|$ is $O\left(|\mathfrak{f} \mathcal{K}|^{2}\right)$, i.e. the transformation of a $\mathfrak{f K B}$ into a classical $\mathrm{KB}$ is quadratic in complexity.

Example 5. Consider the $\mathfrak{H K B}$ of Example 3. We have already shown an excerpt of its reduction into $\mathcal{A L C H}$ during this section. Due to space limitations, the whole reduction of $\mathfrak{f} \mathcal{K}$ cannot be represented in this paper. However, we have seen that $\mathfrak{f} \mathcal{K} \models\langle\alpha \geq 0.5\rangle$, which means that the $\mathfrak{f K B} \mathfrak{f} \mathcal{K}^{\prime}=\langle\mathcal{T}, \mathcal{A} \cup\{\langle\alpha<0.5\rangle\}\rangle$ is not satisfiable. Let us verify that indeed our reduction is satisfiability preserving, by verifying that $\mathcal{K}\left(\mathfrak{f} \mathcal{K}^{\prime}\right)$ is not satisfiable as well. First, let us note that $\sigma(\langle\alpha<0.5\rangle)$ is the assertion

$$
\sigma(\langle\alpha<0.5\rangle)=a: A_{<0.5} \sqcap \forall R_{\geq 0.5} \cdot\left(B_{<0.5} \sqcup C_{<0.5}\right) .
$$

We proceed similarly as for Example 3 . We show that any model $\mathcal{I}$ satisfying $\mathcal{K}\left(\mathfrak{f} \mathcal{K}^{\prime}\right)$, where (11) has been removed, does not satisfy (11). Therefore, there cannot be any model of $\mathcal{K}\left(\mathfrak{f} \mathcal{K}^{\prime}\right)$. Indeed, as $A_{\geq 0.5} \sqcap A_{<0.5} \sqsubseteq \perp$ and $\top \sqsubseteq A_{\geq 0.5} \sqcup A_{<0.5}$ occur in the terminology of $\mathcal{K}\left(\mathfrak{f} \mathcal{K}^{\prime}\right)$, we have that either $a^{\mathcal{I}}$ is an instance of $\left(A_{\geq 0.5}\right)^{\mathcal{I}}$ or $a^{\mathcal{I}}$ is an instance of $\left(A_{<0.5}\right)^{\mathcal{I}}$. In the former case, $\mathcal{I}$ does not satisfy (11). In the latter case, we note that $\forall R . \neg B \sqsubseteq A$ belongs to $\mathcal{T}$ and, thus, $\rho(A,<0.5) \sqsubseteq \rho(\forall R . \neg B,<0.5)$, i.e. $\left.A_{<0.5} \sqsubseteq \exists R_{>0.5} . \bar{B}_{>0.5}\right)$, belongs to the terminology of $\mathcal{K}\left(\mathfrak{f} \overline{\mathcal{K}}^{\prime}\right)$. Therefore, as $a^{\mathcal{I}}$ is an instance of $\left(A_{<0.5}\right)^{\mathcal{I}}, a^{\mathcal{I}}$ has an $\left(R_{>0.5}\right)^{\mathcal{I}}$ successor $d$ which is an instance of $\left(B_{>0.5}\right)^{\mathcal{I}}$. But then, as $\langle a: \forall R . C \geq 0.7\rangle$ occurs in $\mathfrak{f} \mathcal{K}$ and, thus, $a: \forall R_{>0.3} \cdot C_{\geq 0.7}$ occurs in $\mathcal{K}\left(\mathfrak{f} \mathcal{K}^{\prime}\right)$, and $R_{>0.5} \sqsubseteq R_{>0.3}$ is axiom of $\mathcal{K}\left(\mathfrak{f} \mathcal{K}^{\prime}\right)$, it follows that $d$ is also an instance of $\left(C_{\geq 0.7}\right)^{\mathcal{I}}$. Now, it can easily verified that $a^{\mathcal{I}}$ cannot be an instance of $\left(\forall R_{\geq 0.5} \cdot\left(B_{<0.5} \sqcup C_{<0.5}\right)\right)^{\mathcal{I}}$ as $a^{\mathcal{I}}$ has an $\left(R_{\geq 0.5}\right)^{\mathcal{I}}$ successor $d\left(\left(R_{>0.5}\right)^{\mathcal{I}} \subseteq\left(R_{\geq 0.5}\right)^{\mathcal{I}}\right)$, which is neither an instance of $\left(B_{<0.5}\right)^{\mathcal{I}}\left(\left(B_{<0.5}\right)^{\mathcal{I}} \cap\left(B_{>0.5}\right)^{\mathcal{I}}=\emptyset\right)$ nor of $\left(C_{<0.5}\right)^{\mathcal{I}}$ $\left(\left(C_{<0.5}\right)^{\mathcal{I}} \cap\left(C_{\geq 0.7}\right)^{\mathcal{I}}=\emptyset\right)$. Therefore, $\mathcal{I}$ does not satisfy $(11)$.

The following satisfiability preserving reduction theorem can be shown.

Theorem 1. Let $\mathfrak{f} \mathcal{K}$ be $\mathfrak{f} K B$. Then $\mathfrak{f} \mathcal{K}$ is satisfiable iff the $\mathcal{A} \mathcal{L C H} K B \mathcal{K}(\mathfrak{f} \mathcal{K})$ is satisfiable.

Theorem 1, together with Equations (4) and (5), gives us also the possibility to reduce the entailment problem in $\mathfrak{f} \mathcal{A} \mathcal{L C H}$, to an entailment problem in $\mathcal{A} \mathcal{L C H}$. Finally, concerning the BTVB problem, Equation (3) solves straightforwardly the case for 'role assertions'. On the other hand, for assertions of the form $a: C$, we have to solve the case of the $g l b$ only, as from it the $l u b$ can derived (see Equation 2). In [27] it has been shown that $g l b(\mathfrak{f} \mathcal{K}, a: C) \in N^{\mathfrak{f} \mathcal{K}}$ and it can be verified that this holds in $\mathfrak{f} \mathcal{A L C H}$ as well. Therefore, by a binary search on $N^{\mathfrak{f} \mathcal{K}}$, the value of $g l b(\mathfrak{f} \mathcal{K}, \alpha)$ can be determined in at most $\log \left|N^{\mathfrak{f K}}\right|$ entailment tests in $\mathfrak{f} \mathcal{A L C H}$ and, thus, entailment tests in $\mathcal{A L C H}$. Therefore, the BTVB problem can be reduced to $\mathcal{A L C H}$ as well. 


\section{Conclusions}

We have presented a reasoning preserving transformation of $\mathfrak{f} \mathcal{A} \mathcal{L C H}$ into classical $\mathcal{A L C H}$, where general terminological axioms are allowed. This gives us immediately a new method to reason in $\mathfrak{f} \mathcal{A} \mathcal{L C H}$ by means of already existing DL systems and its use in the context of logic-based multimedia information retrieval and, more generally, in the Semantic Web.

Our primary line of future work consists in exploring to which extent the translation technique can be applied to more expressive DLs than $\mathfrak{f} \mathcal{A L C H}$ and contexts where the "truth space" is more general than [0,1], as, for instance, in [28].

\section{References}

1. F. Baader, D. Calvanese, D. McGuinness, D. Nardi, and P. F. Patel-Schneider, editors. The Description Logic Handbook: Theory, Implementation, and Applications. Cambridge University Press, 2003.

2. R. A. Baeza-Yates, R. Baeza-Yates, and B. Ribeiro-Neto. Modern Information Retrieval. Addison-Wesley Longman Publishing Co., Inc., 1999.

3. B. Beckert, R. Hähnle, and F. Manyá. Transformations between signed and classical clause logic. In ISMVL, pages 248-255, 1999.

4. R. Bejar, R. Hahnle, and F. Manyá. A modular reduction of regular logic to classical logic. In $I S M V L$, pages 221-226, 2001.

5. T. Berners-Lee, J. Hendler, and O. Lassila. The semantic web. The Scientifi c American, 284(5):34-43, 2001.

6. G. Bordogna, P Carrara, and G. Pasi. Query term weights as constraints in fuzzy information retrieval. Information Processing and Management, 27(1):15-26, 1991.

7. R. M. da Silva, A. E. C. Pereira, and M. A. Netto. A system of knowledge representation based on formulae of predicate calculus whose variables are annotated by expressions of a fuzzy terminological logic. In IPMU, LNCS 945, Springer-Verlag, 1994.

8. S. Dill, N. Eiron, D. Gibson, D. Gruhl, R. Guha, A. Jhingran, T. Kanungo, S. Rajagopalan, A. Tomkins, J.A. Tomlin, and J.Y. Zien. SemTag: and Seeker: Bootstrapping the semantic web via automated semantic annotation. In $A C M W W W$, Budapest, Hungary, 2003.

9. Th. Gevers and A.W.M. Smeulders. Content-based image retrieval: An overview. In G. Medioni and S. B. Kang, editors, Emerging Topics in Computer Vision. Prentice Hall, 2004.

10. T. R. Giugno and T. Lukasiewicz. P-SHOQ(D): A probabilistic extension of SHOQ(D) for probabilistic ontologies in the semantic web. In JELIA, LNAI 2424, Springer-Verlag, 2002.

11. J. Heinsohn. Probabilistic description logics. In Proc. of the 10th Conference on Uncertainty in Artifi cial Intelligence, pages 311-318, 1994.

12. S. Hölldobler, T. D. Khang, and H.-P. Störr. A fuzzy description logic with hedges as concept modifi ers. In Nguyen Hoang Phuong, Hung T. Nguyen, Nguyen Cat Ho, and Pratit Santiprabhob, editors, Proceedings InTech/VJFuzzy'2002, pages 25-34, Hanoi, Vietnam, 2002. Institute of Information Technology, Vietnam Center for Natural Science and Technology, Science and Technics Publishing House, Hanoi, Vietnam.

13. B. Hollunder. An alternative proof method for possibilistic logic and its application to terminological logics. In 10th Conference on Uncertainty in Artifi cial Intelligence, pages 327335, 1994. Morgan Kaufmann.

14. I. Horrocks, P. F. Patel-Schneider, and F. van Harmelen. From SHIQ and RDF to OWL: The making of a web ontology language. Journal of Web Semantics, 1(1):7-26, 2003. 
15. M. Jäger. Probabilistic reasoning in terminological logics. In Proceedings of KR-94, 5-th International Conference on Principles of Knowledge Representation and Reasoning, pages 305-316, 1994.

16. E.E. Kerre, R.B. Zenner, and R.M. De Caluwe. The use of fuzzy set theory in information retrieval and databases: a survey. Journal of the American Society for Information Science, 37(5):341-345, 1986.

17. M. Kifer and V. S. Subrahmanian. Theory of generalized annotated logic programming and its applications. Journal of Logic Programming, 12:335-367, 1992.

18. D. Koller, A. Levy, and A. Pfeffer. P-CLASSIC: A tractable probabilistic description logic. In Proc. of the 14th Nat. Conf. on Artifi cial Intelligence (AAAI-97), pages 390-397, 1997.

19. D. H. Kraft and D. Buel. Fuzzy sets and generalised boolean retrieval systems. Int. J. ManMachine Studies, 19:45-56, 1983.

20. L. V.S. Lakshmanan and N. Shiri. A parametric approach to deductive databases with uncertainty. IEEE Transactions on Knowledge and Data Engineering, 13(4):554-570, 2001.

21. R. C. T. Lee. Fuzzy logic and the resolution principle. Journal of the ACM, 19(1):109-119, January 1972.

22. C. Lutz, F. Wolter, and M. Zakharyaschev. A tableau algorithm for reasoning about concepts and similarity. In TABLEAUX, LNAI 2796, Springer Verlag, 2003.

23. C. Meghini, F. Sebastiani, and U. Straccia. A model of multimedia information retrieval. Journal of the ACM, 48(5):909-970, 2001.

24. C.V. Negoita and P. Flondor. On fuzziness in information retrieval. Int. J. Man-Machine Studies, 8:711-716, 1976.

25. F. Sebastiani. A probabilistic terminological logic for modelling information retrieval. In Proc. of SIGIR-94, 17th ACM International Conference on Research and Development in Information Retrieval, pages 122-130, Dublin, IRL, 1994. Published by Springer Verlag, Heidelberg, FRG.

26. U. Straccia. A framework for the retrieval of multimedia objects based on four-valued fuzzy description logics. In F. Crestani and Gabriella Pasi, editors, Soft Computing in Information Retrieval: Techniques and Applications, pages 332-357. Physica Verlag (Springer Verlag), Heidelberg, Germany, 2000.

27. U. Straccia. Reasoning within fuzzy description logics. Journal of Artificial Intelligence Research, 14:137-166, 2001.

28. U. Straccia. Uncertainty in description logics: a lattice-based approach. In Proc. IPMU, 2004.

29. C. Tresp and R. Molitor. A description logic for vague knowledge. In Proc. of the 13th European Conf. on Artifi cial Intelligence (ECAI-98), Brighton (England), August 1998.

30. J. Yen. Generalizing term subsumption languages to fuzzy logic. In Proc. of the 12th International Joint Conference on Artifi cial Intelligence (IJCAI-91), pages 472-477, Sydney, Australia, 1991.

31. L. A. Zadeh. Fuzzy sets. Information and Control, 8(3):338-353, 1965. 